\title{
Survival Rates Following Skeletal Metastases-A Twenty-Year Analysis
}

\author{
Mohannad B. Ammori', Sunil Panchani2, Jonathan J. Gregory², James Wylie ${ }^{3}$, Ashok Paul² \\ ${ }^{1}$ Department of Orthopaedic Surgery, Royal Gwent Hospital, Newport, UK \\ ${ }^{2}$ Department of Orthopaedic Surgery, Manchester Royal Infirmary, Manchester, UK \\ ${ }^{3}$ The Christie Hospital, Manchester, UK \\ Email: Mohannad.ammori@doctors.org.uk, sunilpanchani@doctors.org.uk, Jonathan.Gregory@cmft.nhs.uk, \\ James.Wylie@christie.nhs.uk, ashok.paul@cmft.nhs.uk
}

Received 13 August 2015; accepted 13 September 2015; published 16 September 2015

Copyright @ 2015 by authors and Scientific Research Publishing Inc.

This work is licensed under the Creative Commons Attribution International License (CC BY).

http://creativecommons.org/licenses/by/4.0/

(c) (i) Open Access

\begin{abstract}
Introduction: The aims of this study were to establish an estimate of the prognosis and to identify the variables predictive of survival following the diagnosis of metastatic bone disease. Materials and Methods: Ninety-seven patients diagnosed with skeletal metastases confirmed by histological examination between 1991 and 2010 were selected. Data were retrospectively collected including age and sex, sites and histology of the primary tumour and skeletal metastases, presence of visceral metastases, pathological fracture, and the treatment received. Results: Ninety-three patients (95.9\%) were deceased. The overall rate of survival was $47 \%$ at 6 months, $31 \%$ at one year and $10 \%$ at three years. Multivariate analysis confirmed the grade of malignancy, age and systemic therapy as independent predictors of survival. Discussion and Conclusion: The prognosis of patients with skeletal metastases should be estimated according to the site and histology of the primary tumour, grade of malignancy and age at the time of presentation.
\end{abstract}

\section{Keywords}

Oncology, Skeletal Metastases, Survival

\section{Introduction}

Skeletal metastases are often indicative of an advanced stage of cancer and generally reflect a poor prognosis. Information available regarding the survival following the diagnosis of metastatic bone disease is variable throughout the literature and focused upon the site of the primary malignancy, the skeletal metastases and a variety of other factors.

\footnotetext{
"Corresponding author.
}

How to cite this paper: Ammori, M.B., Panchani, S., Gregory, J.J., Wylie, J. and Paul, A. (2015) Survival Rates Following Skeletal Metastases-A Twenty-Year Analysis. Open Journal of Orthopedics, 5, 288-296. 
Previous studies have identified the site of the primary malignancy, presence of visceral metastases and the complication of a pathological fracture as independent predictors of survival [1]-[5]. Furthermore, elevated bone-specific alkaline phosphatase (ALP), haemoglobin ( $\mathrm{Hb}$ ) of less than $7 \mathrm{mmol} / \mathrm{L}$ and hypercalcaemia have been identified as negative prognostic factors [1] [6]. Surgery, radiotherapy and systemic therapy have proven beneficial in the management of patients with metastatic bone disease [4] [7] [8]. Operative intervention and radiotherapy are often palliative procedures. The aim of surgery is to achieve local control of the metastasis as well as structural stability in order to restore function as quickly as possible [7]. Surgical intervention should be considered in patients with an impending or actual pathological fracture and for the management of pain on mobilisation or with the activities of daily living. For limb lesions, surgical options include stabilisation or endoprosthetic reconstruction. Stabilisation, de-bulking and laminectomy are undertaken in patients with vertebral metastases. The prognosis of patients with skeletal metastases is important in guiding the choice of operative intervention. Endoprosthetic replacement is recommended for patients with a relatively good prognosis, as the use of internal fixation is associated with a high risk of implant failure [9]. The use of therapeutic agents is dependent upon the histology of the primary malignancy, and therefore it is important to note that skeletal metastases most commonly originate from tumours of the prostate, breast, kidney and lung [10].

The aims of this retrospective study were to identify the variables predictive of survival following the diagnosis of skeletal metastases and to establish an estimate of the prognosis in order to plan treatment.

\section{Materials and Methods}

\subsection{Patients}

Ninety-seven patients with a histological diagnosis of skeletal metastases between 1991 and 2010 were selected. A total of 156 patients were identified from the pathology database; 59 patients were excluded due to the absence of a positive diagnosis of skeletal metastases, the diagnosis of multiple myeloma, Waldenström's macroglobulinaemia and the absence of information due to the destruction of hospital records. Data were retrospectively collected from digital databases (Patient Admin System (PAS), Clinical Work Station (CWS), Patient Archiving Communication System (PACS), Medisec) and the hospital records of two separate institutions. The information included patient demographics such as age and sex; sites and histology of the primary tumour and skeletal metastases; presence of visceral metastases; pathological fracture; and the treatment received. Data collection was undertaken in accordance with the ethical standards and approval of the University of Manchester Research Ethics Committee. The details of the patients are summarised in Table 1.

The primary tumours were classified into three groups according to the grade of malignancy: 1) high (lung, oesophagus, stomach, pancreas, liver, gallbladder and leiomyosarcoma), 2) intermediate (colon, kidney, bladder, ovary, endometrium, cervix, malignant melanoma, merkel cell carcinoma and unknown), and 3) low (thyroid, breast, prostate and lymphoma). This was determined in part from examples of similar classification systems within the literature [1] [3]. The duration of survival was defined from the date of histological or radiological diagnosis of skeletal metastases to the date of death or latest follow-up.

The incidence of metastases to each skeletal component and the percentage of consequential pathological fracture are illustrated in Figure 1. Skeletal metastases most commonly affected the vertebrae, femur, pelvis and ribs.

\subsection{Management}

Skeletal metastases were treated with surgical intervention, radiotherapy or systemic therapy (chemotherapy, hormone and immune therapy). Patients who were at risk of or who had sustained a pathological fracture within the appendicular skeleton received internal fixation or prosthetic replacement. Surgical intervention in the form of fixation, de-bulking and laminectomy was reserved for patients with skeletal metastases to the vertebral component of the axial skeleton. Two patients received embolisation of skeletal metastases: one with a primary diagnosis of renal cell carcinoma and the other with thyroid adenocarcinoma. Radiotherapy was not usually used as the sole treatment of skeletal metastases and was most often used post-operatively. The administration of systemic therapy was dependent upon the site and histology of the primary tumour. The most common primary malignancies were pulmonary $(35.1 \%)$, renal $(12.4 \%)$, breast $(12.4 \%)$ and prostate $(11.3 \%)$ carcinoma. Contemporary chemotherapy was provided at the supra-regional oncology centre. Patients who failed to respond to standard regimes were considered for entry in clinical trials where appropriate. 


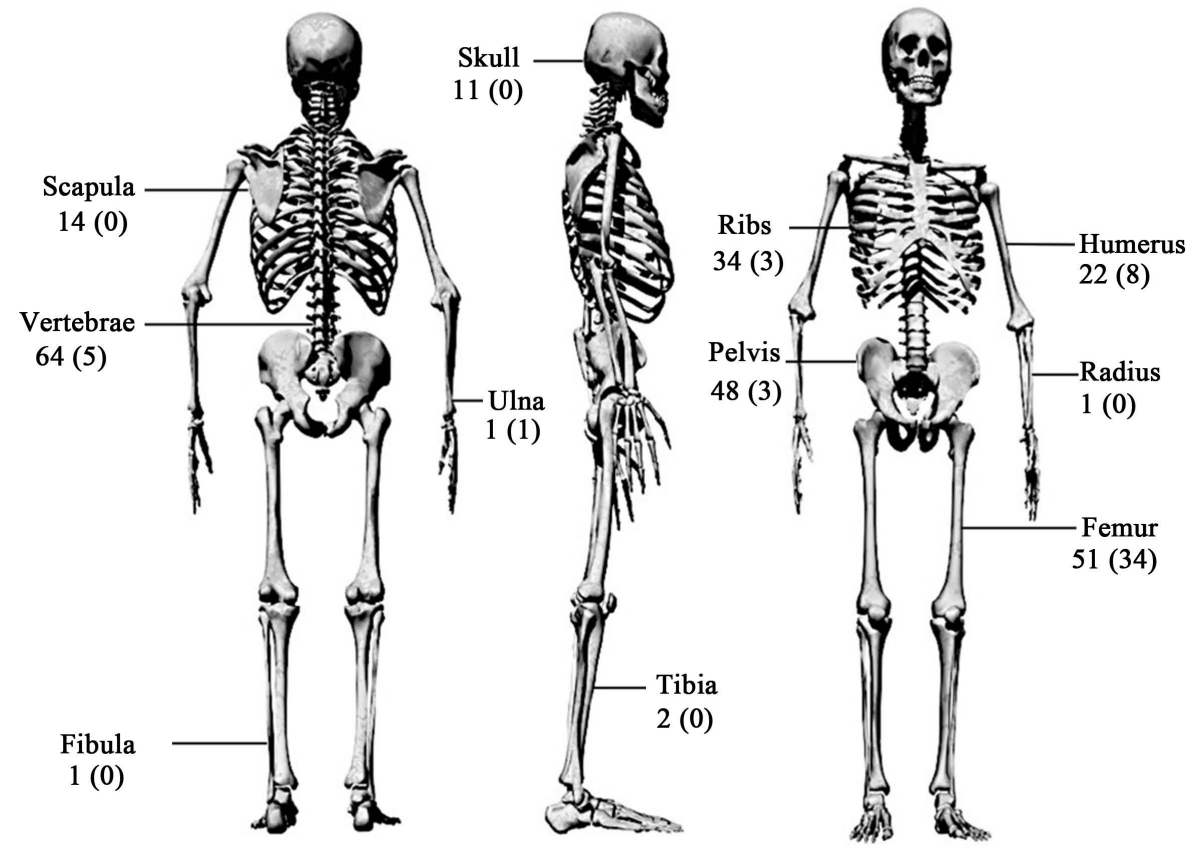

Figure 1. Incidence of metastases and pathological fracture to each skeletal component. Data shown represent number of patients with metastases (pathological fracture), $\mathrm{n}=97$.

Table 1. Summary of patient details.

\begin{tabular}{|c|c|c|}
\hline & & Total \\
\hline Number of patients & & $97(100.0 \%)$ \\
\hline Age (years) ${ }^{*}$ & & $65.4(36.7 \%-92.1 \%)$ \\
\hline \multirow{3}{*}{ Sex } & Male & $57(58.8 \%)$ \\
\hline & Female & $40(41.2 \%)$ \\
\hline & High & $43(44.3 \%)$ \\
\hline \multirow[t]{2}{*}{ Grade of malignancy } & Intermediate & $28(28.9 \%)$ \\
\hline & Low & $26(26.8 \%)$ \\
\hline \multirow{3}{*}{ Visceral metastases } & Yes & $44(45.4 \%)$ \\
\hline & No & $53(54.6 \%)$ \\
\hline & Yes & $48(49.5 \%)$ \\
\hline \multirow[t]{3}{*}{ Initial presentation of metastases } & No & $44(45.3 \%)$ \\
\hline & Unknown & $5(5.2 \%)$ \\
\hline & One & $16(16.5 \%)$ \\
\hline \multirow[t]{2}{*}{ Number of metastases } & Two & $6(6.2 \%)$ \\
\hline & Multiple & $75(77.3 \%)$ \\
\hline \multirow{3}{*}{ Pathological fracture } & Yes & $54(55.7 \%)$ \\
\hline & No & $43(44.3 \%)$ \\
\hline & Surgery & $48(49.5 \%)$ \\
\hline \multirow[t]{2}{*}{ Treatment } & Radiotherapy & $72(74.2 \%)$ \\
\hline & Systemic & $40(41.2 \%)$ \\
\hline \multirow{2}{*}{ Deceased } & Yes & $93(95.9 \%)$ \\
\hline & No & $4(4.1 \%)$ \\
\hline \multicolumn{2}{|c|}{ Survival (months) ${ }^{*}$} & $5.7(0.2-142.1)$ \\
\hline
\end{tabular}

Data shown represent number (percentage); ${ }^{*}$ Data shown represent median (range). 


\subsection{Statistical Analysis}

We analysed the data using the software package SPSS 19 (Chicago, Illinois, USA). The results were expressed as median or percentage as appropriate. A Cox proportional hazards regression was applied to determine dependent and independent predictors of survival. Variables identified with a $p$-value of $<0.2$ were included in the Cox regression multivariate analysis. Significance was accepted at $p<0.05$. Kaplan-Meier survival analysis was undertaken to illustrate the duration of survival.

\section{Results}

\subsection{Survival}

The median survival was 5.7 (range 0.2 - 142.1) months. Ninety-three patients $(95.9 \%)$ were deceased and the remaining four (4.1\%) received follow-up at a median (range) duration of 27.7 (16.7 - 92.4) months. The overall rate of survival was $47 \%$ at 6 months, $31 \%$ at one year and $10 \%$ at three years. A Kaplan-Meier curve demonstrating survival following the diagnosis of skeletal metastases is displayed in Figure 2. Cohorts of patients with renal cell or prostatic carcinoma had amongst the best median (mean, range) survival of $19.4(32.7,1.5$ - 119.6$)$ and 16.7 (24.4, 0.2 - 63.2) months respectively; those who suffered from breast carcinoma survived for a median (mean, range) period of $9.7(29.0,1.7-142.1)$ months. The cohort of patients with carcinoma of the lung experienced a relatively short median (mean, range) survival of $3.7(5.6,0.5-27.7)$ months.

\subsection{Factors Predictive of Survival}

A Cox proportional hazards regression analysis of the factors that could potentially influence survival was undertaken. The variables included in the analysis were age, sex, grade of primary malignancy, presence of visceral metastases, number of metastases, initial presentation, pathological fracture and treatment of skeletal metastases in the form of surgery and systemic therapy. Surgical intervention was also not included in the multivariate analysis, as surgery would usually be offered only to patients who were expected to survive for longer than six weeks. Univariate analysis identified the grade of the primary malignancy, initial diagnosis of skeletal metastases, surgery and systemic therapy to be dependent predictors of survival (Table 2). Multivariate analysis confirmed the grade of malignancy of the primary tumour and systemic therapy to be independent predictors' survival.

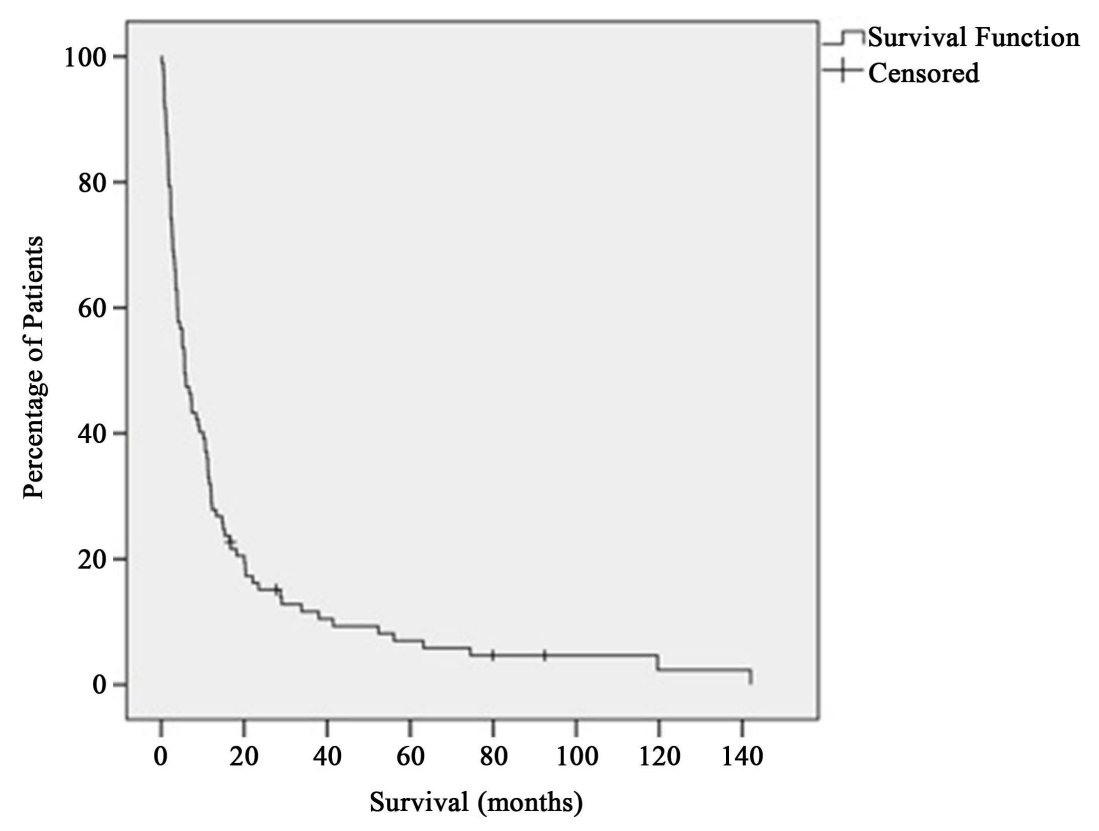

Figure 2. Kaplan-Meier curve demonstrating survival following the diagnosis of skeletal metastases. 
Table 2. Factors predictive of survival in patients with skeletal metastases $(n=97)$.

\begin{tabular}{|c|c|c|c|c|c|c|}
\hline \multirow{2}{*}{ Variable } & \multicolumn{3}{|c|}{ Univariate } & \multicolumn{3}{|c|}{ Multivariate } \\
\hline & $p$ value & HR & $95 \% \mathrm{CI}$ & $p$ value & HR & $95 \%$ CI \\
\hline Age & 0.741 & 0.997 & $0.979-1.016$ & & & \\
\hline Sex & 0.773 & 1.063 & $0.701-1.612$ & & & \\
\hline \multicolumn{7}{|l|}{ Grade of malignancy } \\
\hline \multicolumn{7}{|l|}{ High (reference group) } \\
\hline Intermediate & 0.003 & 0.457 & $0.273-0.764$ & 0.011 & 0.478 & $0.271-0.844$ \\
\hline Low & $<0.0001$ & 0.306 & $0.175-0.533$ & 0.011 & 0.420 & $0.215-0.822$ \\
\hline Visceral metastases & 0.481 & 1.162 & $0.766-1.763$ & & & \\
\hline \multicolumn{7}{|l|}{ Number of metastases } \\
\hline \multicolumn{7}{|l|}{ Single (reference group) } \\
\hline Two & 0.937 & 0.962 & $0.369-2.507$ & & & \\
\hline Multiple & 0.367 & 1.298 & $0.737-2.286$ & & & \\
\hline Presentation of metastases & 0.014 & 1.741 & $1.116-2.715$ & 0.314 & 1.299 & $0.780-2.164$ \\
\hline Pathological fracture & 0.728 & 1.077 & $0.710-1.634$ & & & \\
\hline Surgery & 0.003 & 0.527 & $0.344-0.808$ & & & \\
\hline Systemic therapy & 0.002 & 0.505 & $0.330-0.772$ & 0.041 & 0.618 & $0.389-0.982$ \\
\hline
\end{tabular}

\subsection{Vertebral Metastases}

A univariate analysis to evaluate whether any of the above factors were predictive of survival in a cohort of 64 patients with vertebral metastases identified the grade of primary malignancy, initial presentation of skeletal metastases, surgery and systemic therapy to have a significant influence (Table 3 ). Only the grade of malignancy of the primary tumour was confirmed to be an independent predictor of survival.

\subsection{Radiotherapy}

A Cox proportional hazards regression analysis of the above factors, which could potentially influence survival in a cohort of 69 patients who received radiotherapy, was undertaken (Table 4). Univariate analysis found the grade of malignancy of the primary tumour, initial presentation of skeletal metastases, surgery and systemic therapy to have a significant influence. Furthermore, age and the presence of visceral metastases qualified for inclusion in the Cox regression multivariate analysis. This identified age, the grade of the primary malignancy and systemic therapy to be independent predictors of survival.

\section{Discussion}

Several predictors of prognosis for patients with skeletal metastases have been reported throughout the literature. Hansen et al. identified pathological fracture, visceral metastases, haemoglobin content $<7 \mathrm{mmol} / \mathrm{L}$ and lung cancer as negative prognostic factors [1]. Katagiri et al. reported the site of the primary tumour, performance status, previous chemotherapy, multiple metastases and the presence of visceral or cerebral metastases to be significant predictors of survival [2]. The absence of a pathological fracture and visceral metastases, a time interval of more than three years between the diagnosis of cancer and the first skeletal metastases, and carcinoma of the thyroid, kidney, prostate and breast were confirmed to be positive prognostic variables [8].

We identified the grade of malignancy and systemic therapy to be significant predictors of prognosis. A subsequent multivariate analysis, which we undertook in a cohort of patients who received radiotherapy, identified younger age, the grade of malignancy and the absence of systemic therapy to be negative predictors of survival. 
Table 3. Factors predictive of survival in patients with vertebral metastases $(n=64)$.

\begin{tabular}{|c|c|c|c|c|c|c|}
\hline \multirow{2}{*}{ Variable } & \multicolumn{3}{|c|}{ Univariate } & \multicolumn{3}{|c|}{ Multivariate } \\
\hline & $p$ value & HR & $95 \%$ CI & $p$ value & HR & $95 \%$ CI \\
\hline Age & 0.701 & 1.004 & $0.983-1.026$ & & & \\
\hline Sex & 0.391 & 1.257 & $0.745-2.119$ & & & \\
\hline \multicolumn{7}{|l|}{ Grade of malignancy } \\
\hline \multicolumn{7}{|l|}{ Low (reference group) } \\
\hline Intermediate & 0.007 & 2.623 & $1.302-5.283$ & 0.026 & 2.345 & $1.108-4.962$ \\
\hline High & $<0.0001$ & 3.784 & $1.894-7.557$ & 0.012 & 2.958 & $1.270-6.891$ \\
\hline Visceral metastases & 0.514 & 1.187 & $0.709-1.988$ & & & \\
\hline \multicolumn{7}{|l|}{ Number of metastases } \\
\hline \multicolumn{7}{|l|}{ Single (reference group) } \\
\hline Two & 0.166 & 2.752 & $0.657-11.524$ & & & \\
\hline Multiple & 0.295 & 1.591 & $0.667-3.793$ & & & \\
\hline Presentation of metastases & 0.046 & 1.752 & $1.009-3.041$ & 0.517 & 1.250 & $0.636-2.457$ \\
\hline Pathological vertebral fracture & 0.645 & 0.787 & $0.283-2.184$ & & & \\
\hline Surgery & 0.003 & 0.164 & $0.049-0.548$ & & & \\
\hline Systemic therapy & 0.007 & 0.484 & $0.285-0.822$ & 0.215 & 0.698 & $0.395-1.232$ \\
\hline
\end{tabular}

Table 4. Factors predictive of survival in patients with skeletal metastases who received radiotherapy $(n=69)$.

\begin{tabular}{|c|c|c|c|c|c|c|}
\hline \multirow{2}{*}{ Variable } & \multicolumn{3}{|c|}{ Univariate } & \multicolumn{3}{|c|}{ Multivariate } \\
\hline & $p$ value & HR & $95 \% \mathrm{CI}$ & $p$ value & HR & $95 \%$ CI \\
\hline Age & 0.122 & 0.982 & $0.960-1.005$ & 0.011 & 0.964 & $0.937-0.992$ \\
\hline Sex & 0.822 & 0.945 & $0.575-1.552$ & & & \\
\hline
\end{tabular}

Grade of malignancy

High (reference group)

Intermediate

Low

Visceral metastases

Number of metastases

Single (reference group)

Two

Presentation of metastases

Pathological fracture

$$
\text { Surgery }
$$

Systemic therapy

\section{$<0.0001$}

$<0.0001$

0.196

0.297

$0.154-0.573$

0.003

$0.107-0.414$

0.010

$0.841-2.333$

0.290

$\begin{array}{ll}0.316 & 0.149-0.671 \\ 0.335 & 0.146-0.769 \\ 1.374 & 0.763-2.476\end{array}$

$\begin{array}{ll}0.335 & 0.146-0.769 \\ 1.374 & 0.763-2.476\end{array}$

0.971

0.242

0.011

0.622

0.022

0.017
0.981

$0.341-2.822$

1.473

1.965

$0.769-2.822$

$1.164-3.316$

1.133

$0.690-1.861$

0.554

$0.335-0.918$

0.544

$0.330-0.898$

0.031

0.531

$0.299-0.943$ 
Several studies have classified the site of the primary tumour according to the grade of malignancy and have found this to be a significant predictor of prognosis [1]-[4] [8]. Katagari et al. reported an overall survival, versus this study, of 0.66 (vs. 0.47) at six months, 0.48 (vs. 0.31) at 12 months and 0.23 (vs. 0.10) at 36 months, respectively [14]. The differences in survival between Katagari et al. and this study reflect the different proportion of patients with low-and high-grade malignancies: low, 0.41 vs. 0.28 ; intermediate 0.21 vs. 0.29 ; high, 0.38 vs. 0.44 , respectively. A greater proportion of the patients in Katageri et al suffered from a low-grade primary malignancy, whilst a greater proportion of patients in this study suffered from a primary malignancy of an intermediate-and high-grade [2]. This discrepancy further supports the influence of the grade of malignancy upon the survival period.

Skeletal metastases most commonly originate from primary tumours of the lung, prostate, breast and kidney [4] [10] [11]. A study identified the survival period following the diagnosis of skeletal metastases originating from carcinoma of the prostate and breast as 24 (vs. 24.4) and 34 (vs. 29.0) months, versus this study, respectively [11]. Furthermore, an analysis of a cohort of patients who received surgical management of metastatic bone disease reported the mean survival following the diagnosis of skeletal metastases secondary to prostate carcinoma as 40.0 (vs. 24.4) months, breast carcinoma as 44.1 (vs. 29.0) months and renal cell carcinoma as 30.6 (vs. 32.7) months [8]. The prognoses of patients with prostate and breast carcinoma were relatively prolonged, however a selection bias was present as this study as it only included patients who were fit enough for surgical intervention. The prognosis of patients with skeletal metastases of pulmonary origin has been reported throughout the literature. Campanacci et al. reported a prognosis of less than twelve months [11]; Katakami et al suggested a prognosis of less than six months [12]. Further studies have suggested a mean survival of approximately six months [13], whilst others report a median of $5.8-10.0$ months [14] [15]. We report an overall mean (median) survival of 5.8 (3.7) months following the diagnosis of skeletal metastases secondary to pulmonary carcinoma.

The influence of the initial presentation of skeletal metastases upon survival was identified as a negative predictor of prognosis on univariate analysis. Patients who present late with metastatic disease would not have received previous treatment. Although the late administration of chemotherapy may be thought to be a negative predictor of survival, studies have found the absence of previous therapeutic administration prior to the diagnosis of skeletal metastases to be a positive prognostic factor [2] [16]. Katagiri et al. explained the previous administration of chemotherapy reflected an advanced stage of cancer, and the lack of further effective chemotherapeutic options following the diagnosis of skeletal metastases [2].

Systemic therapy was reported to be independently associated with a positive prognosis. However, the significance of this variable should be interpreted with caution, as a selection bias may be responsible. Dependent upon the primary malignancy, some chemotherapeutic agents were known to be ineffective in the treatment of skeletal metastases and thus were not administered. Studies have reported the administration of systemic therapeutic agents as positive predictors of prognosis in patients with metastatic bone disease [17] [18]. Wibmer et al. found systemic therapy to be an independent positive prognostic variable in patients with vertebral metastases [4]. We identified surgery and systemic therapy as dependent predictors of prognosis within the same cohort. A comparative study evaluated the effectiveness of surgical intervention and radiotherapy against radiotherapy alone and found a significant proportion of patients were able to walk following operative intervention, reflecting the efficacy of surgery as opposed to radiotherapy alone in the treatment of vertebral metastases [7]. Mizumoto et al. reported a median survival of 6.2 months in a cohort of patients who primarily received radiotherapy for metastases to the vertebral column [16]. An alternative study of patients who primarily received surgical intervention and systemic therapy reported a median survival of 10.6 months [4]. These studies support the efficacy of surgical intervention and systemic therapy as opposed to radiotherapy in the treatment of patients with vertebral metastases.

However, the choice of treatment is influenced by the prognosis of the patient, and therefore the Oswestry Spinal Risk Index and the Tokuhashi score have been developed in an attempt to predict the period of survival according to the primary malignancy and the patient condition [19] [20]. The efficacy of radiotherapy may be dependent upon the primary malignancy. A recent study reported patients with breast cancer were significantly more likely to achieve good local control with radiotherapy to the vertebral column [16]. Chow et al. compared the administration of single- and multiple-fraction schedules of radiotherapy, and reported no significant difference in the rate of response [21]. Fixation should be considered as a prophylactic intervention to prevent the pain and loss of function consequential to a pathological fracture. Elective stabilisation was reported to be associated 
with a shorter hospital stay and an increased likelihood of being discharged home as opposed to a nursing home or rehabilitation facility [3].

We reported an incidence of pathological fracture of the femur or humerus to be $41.2 \%$, considerably higher than the $8 \%$ reported in the literature [22]. This discrepancy may be due to a selection bias in our study, as patients who experience a pathological fracture are more likely to receive a histological confirmation following surgery. Huber et al. found a pathological fracture to be a negative predictor of prognosis [8]. We report that this complication did not influence survival, supporting the statements of Katagari et al. and Wibmer et al. Coleman et al. suggested the probability of developing a pathological fracture increases with the duration of metastatic bone disease [22]. Although the diagnosis of a pathological fracture may indicate an advanced stage of metastatic bone disease, prevalence in patients with a relatively good prognosis eliminates the significance of this variable in a statistical evaluation.

The variables predictive of survival should be taken into consideration in the choice of surgical management. Patient selection for operative fixation should be dependent upon the expected prognosis, as patients could otherwise be subject to implant failure. Patients with a low-grade primary tumour or a high grade primary malignancy with a low volume of metastatic disease are likely to have a greater life expectancy such that internal fixation is not recommended as patients are likely to outlive the construct and endoprosthetic replacement is the preferred operative intervention [23] [24]. Wedin et al. reported a notably greater failure rate of internal fixation with osteosynthetic devices compared with endoprosthetic reconstruction for proximal femoral metastases [20] However, endoprosthetic reconstruction involves a more extensive operation and may not be appropriate for patients with a poor prognosis. Patients with a pathological fracture following skeletal metastases from a high-grade primary malignancy with a high volume of metastatic disease should be considered for internal fixation, as the expected prognosis is often poor. However, implants, which only provide relative stability, may not achieve good analgesia as the bone, which has been invaded by tumour, often has little mechanical integrity and the fracture is unlikely to unite. The aim of operative fixation should be to achieve stabilisation of the fracture and resist the cyclical load at this un-united site for the duration of the patient's survival. Locking plates are known to provide a reliable form of fixation and excellent functional restoration in peri-articular skeletal metastases, and polymethylmethacrylate augmentation has been reported to reduce pain and to provide a durable construct [24].

This study provides a comprehensive overview of the survival of patients who suffered from skeletal metastases secondary to a variety of different primary malignancies, which reflects the nature of the presentation of this pathology to an Orthopaedic surgeon. Information regarding the variables predictive of survival and an estimate of the prognosis aids the clinician in the choice of surgical intervention. The limitations of this study include the retrospective data collection for a prolonged period of twenty years and a selection bias, as patients who sustained a pathological fracture are more likely to meet the inclusion criteria of a histological diagnosis of skeletal metastases. Systemic therapy would not be appropriate or effective for every primary malignancy, and surgical intervention would only usually be offered to patients who were expected to survive for at least six weeks.

\section{Conclusion}

The prognosis of patients with skeletal metastases should be estimated according to the site and histology of the primary tumour. A high grade of malignancy and a younger age at the time of presentation are negative predictors of prognosis. The results of this study are comparable to and supportive of the current literature. The association of systemic therapy with a prolonged survival should be interpreted with caution, as treatment was subject to a selection bias.

\section{Conflicts of Interest}

The authors have no conflicts of interest or financial ties to disclose.

\section{References}

[1] Hansen, B.H., Keller, J., Laitinen, M., et al. (2004) The Scandinavian Sarcoma Group Skeletal Metastasis Registry. Survival after Surgery for Bone Metastasis in the Pelvis and Extremities. Acta Orthopaedica Scandinavica, 75, 11-15. http://dx.doi.org/10.1080/00016470410001708270

[2] Katagiri, H., Takahashi, M., Wakai, K., et al. (2005) Prognostic Factors and a Scoring System for Patients with Skele- 
tal Metastasis. The Journal of Bone \& Joint Surgery (British), 87, 698-703. http://dx.doi.org/10.1302/0301-620X.87B5.15185

[3] Tomita, K., Kawahara, N., Kobayashi, T., et al. (2001) Surgical Strategy for Spinal Metastases. Spine, 26, 298-306. http://dx.doi.org/10.1097/00007632-200102010-00016

[4] Wibmer, C., Leithner, A., Hofmann, G., et al. (2011) Survival Analysis of 254 Patients after Manifestation of Spinal Metastases-Evaluation of Seven Preoperative Scoring Systems. Spine, 36, 1977-1986. http://dx.doi.org/10.1097/BRS.0b013e3182011f84

[5] Yong, M., Jensen, A.O., Jacobsen, J.B., et al. (2011) Survival in Breast Cancer Patients with Bone Metastases and Skeletal-Related Events: A Population-Based Cohort Study in Denmark (1999-2007). Breast Cancer Research and Treatment, 129, 495-503. http://dx.doi.org/10.1007/s10549-011-1475-5

[6] Brown, J.E., Cook, R.J., Major, P., et al. (2005) Bone Turnover Markers as Predictors of Skeletal Complications in Prostate Cancer, Lung Cancer and Other Solid Tumours. Journal of the National Cancer Institute, 97, 59-69. http://dx.doi.org/10.1093/jnci/dji002

[7] Bickels, J., Dadia, S. and Lidar, Z. (2009) Surgical Management of Metastatic Bone Disease. The Journal of Bone \& Joint Surgery (American), 91, 1503-1516. http://dx.doi.org/10.2106/JBJS.H.00175

[8] Böhm, P. and Huber, J. (2002) The Surgical Treatment of Bony Metastases of the Spine and Limbs. The Journal of Bone \& Joint Surgery (British), 84, 521-529. http://dx.doi.org/10.1302/0301-620X.84B4.12495

[9] Wedin, R., Bauer, H.C. and Rutqvist, L.E. (2001) Surgical Treatment for Skeletal Breast Cancer Metastases: A Population-Based Study of 641 Patients. Cancer, 92, 257-262. http://dx.doi.org/10.1002/1097-0142(20010715)92:2<257::AID-CNCR1317>3.0.CO;2-R

[10] British Orthopaedic Association and the British Orthopaedic Oncology Society. Metastatic Bone Disease: A Good Guide to Practice.

http://www.spitalmures.ro/_files/protocoale terapeutice/oncologie/metastatic bone disease.pdf

[11] Campanacci, M. (1999) Bone and Soft Tissue Tumours. 2nd Edition, Springer-Vaerlag, New York. http://dx.doi.org/10.1007/978-3-7091-3846-5

[12] Katakami, N. (2006) Lung Cancer with Bone Metastasis. Gan to kagaku ryoho, 33, 1049-1053.

[13] Ando, M., Ando, Y., Sugiura, S., et al. (1999) Prognostic Factors for Short-Term Survival in Patients with Stage IV Non-Small Cell Lung Cancer. Japanese Journal of Cancer Research, 90, 249-253. http://dx.doi.org/10.1111/j.1349-7006.1999.tb00740.x

[14] Decroisette, C., Monnet, I., Berard, H., et al. (2011) Epidemiology and Treatment Costs of Bone Metastases from Lung Cancer: A French Prospective, Observational, Multicenter Study (GFPC 0601). Journal of Thoracic Oncology, 6, 576582. http://dx.doi.org/10.1097/JTO.0b013e318206a1e3

[15] Peng, H., Ma, M. and Han, B. (2011) Survival Analysis of 1,742 Patients with Stage IV Non-Small Cell Lung Cancer. Chinese Journal of Lung Cancer, 14, 362-366.

[16] Mizumoto, M., Harada, H., Asakura, H., et al. (2011) Radiotherapy for Patients with Metastases to the Spinal Column: A Review of 603 Patients at Shizuoka Cancer Centre Hospital. International Journal of Radiation Oncology*Biology* Physics, 79, 208-213. http://dx.doi.org/10.1016/j.ijrobp.2009.10.056

[17] Lee, R.J., Saylor, P.J. and Smith, M.R. (2010) Contemporary Therapeutic Approaches Targeting Bone Complications in Prostate Cancer. Clinical Genitourinary Cancer, 8, 29-36. http://dx.doi.org/10.3816/CGC.2010.n.005

[18] Yuasa, T., Urakami, S., Yamamoto, S., et al. (2011) Treatment Outcome and Prognostic Factors in Renal Cell Cancer Patients with Bone Metastasis. Clinical \& Experimental Metastasis, 28, 405-411. http://dx.doi.org/10.1007/s10585-011-9379-7

[19] Balain, B., Jaiswal, A., Trivedi, J.M., et al. (2013) The Oswestry Risk Index: An Aid in the Treatment of Metastatic Disease of the Spine. The Bone \& Joint Journal, 95, 210-216. http://dx.doi.org/10.1302/0301-620X.95B2.29323

[20] Tokuhashi, Y., Matsuzaki, H., Oda, H., et al. (2005) A Revised Scoring System for Preoperative Evaluation of Metastatic Spine Tumor Prognosis. Spine, 30, 2186-2191. http://dx.doi.org/10.1097/01.brs.0000180401.06919.a5

[21] Chow, E., Harris, K., Fan, G., et al. (2007) Palliative Radiotherapy Trials for Bone Metastases: A Systematic Review. Journal of Clinical Oncology, 25, 1423-1436. http://dx.doi.org/10.1200/JCO.2006.09.5281

[22] Coleman, R.E. (1997) Skeletal Complications of Malignancy. Cancer, 80, 1588-1594. http://dx.doi.org/10.1002/(SICI)1097-0142(19971015)80:8+<1588::AID-CNCR9>3.0.CO;2-G

[23] Gregory, J.J. and Cool, P. (2012) Skeletal Metastases and Pathological Fractures. In: Sivananthan, S., Sherry, E., Warnke, P. and Miller, M., Eds., Mercer's Textbook of Orthopaedics and Trauma, 10th Edition, Hodder Arnold, London, 925-939. http://dx.doi.org/10.1201/b13543-95

[24] Gregory, J.J., Ockendon, M., Cribb, G.L., et al. (2011) The Outcome of Locking Plate Fixation for the Treatment of Periarticular Metastases. Acta Orthopcedica Belgica, 77, 362-370. 